COSTA RICAN UNIVERSITY ENTREPRENEURIAL ECOSYSTEM AND ITS LINK WITH THE ENTREPRENEURIAL INTENTION: AN EXPLORATORY STUDY

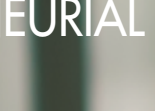


The literature on entrepreneurial university ecosystems has being evidence of possible factors related to entrepreneurial intention, however, there is still a significant information gap. This article seeks to provide a conceptual model that integrates various components of a university entrepreneurial ecosystem that could be related to entrepreneurial intention. The qualitative exploratory study, based on 10 in-depth interviews with professionals linked to entrepreneurial activity in 10 Costa Rican universities, public and private, proposes six pillars: curriculum design, co-curricular activities, organizational infrastructure, strategic commitment, the allocation of financial resources, and the participation of global academic and entrepreneurship networks. It defines, also, technology as a transversal axis. The study confirms, from the point of view of the interviewees, that a link may exists between a set of pillars and their components with the entrepreneurial intention. It highlights the strategic alignment of the universities, the promotion of an entrepreneurial culture, the value of co-curricular activities, the teaching role and the articulation with the national ecosystem.

KEYWORDS: University entrepreneurship, entrepreneurial ecosystem, entrepreneurial intention

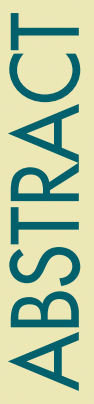

\section{Daniela Herrera-Valverde}

Estudiante de posgrado. Escuela de Administración de Empresas, Instituto Tecnológico de Costa Rica, Campus Cartago, Costa Rica.

danielah@estudiantec.cr

\section{Ronald Mora-Esquivel}

Profesor. Escuela de Administración de Empresas, Instituto Tecnológico de Costa Rica, Campus Cartago, Costa Rica.

rmora@itcr.ac.cr

Juan Carlos Leiva emprendedores ha evidenciado posibles factores relativos a la intención emprendedora, sin embargo, todavía existe un vacío significativo de información. Este artículo busca aportar un modelo conceptual que integre diversos componentes de un ecosistema emprendedor universitario que podrían estar asociados a la intención emprendedora. El estudio cualitativo, de alcance exploratorio, basado en diez entrevistas en profundidad a profesionales ligados con la actividad emprendedora en diez universidades, públicas y privadas de Costa Rica, plantea seis pilares: el diseño curricular, las actividades co-curriculares, la infraestructura organizativa, el compromiso estratégico, la asignación de recursos financieros y la participación de redes globales académicas y de emprendimiento. Asimismo, define la tecnología como eje transversal. El estudio confirma, desde la óptica de los entrevistados, un posible conjunto de pilares y sus componentes que estarían asociados con la intención emprendedora. Se destaca la importancia del alineamiento estratégico de las universidades, la promoción de una cultura emprendedora, el valor de las actividades co-curriculares, el rol docente y la articulación con el ecosistema nacional.

PALABRAS CLAVE: Emprendimiento universitario, ecosistema emprendedor, intención emprendedora.

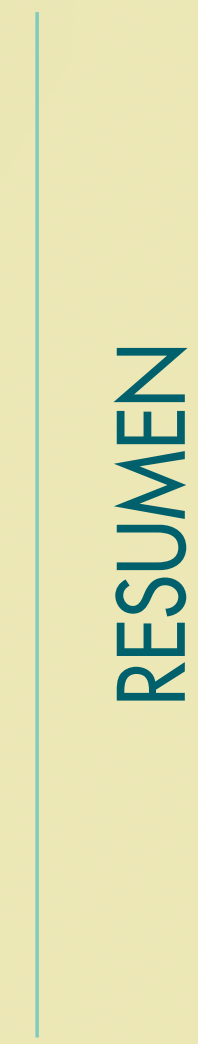

Profesor. Escuela de Administración de Empresas, Instituto Tecnológico de Costa Rica, Campus Cartago, Costa Rica

¡leiva@itcr.ac.cr

ARTÍCULO RECIBIDO:

20/11/2019

ARTÍCULO ACEPTADO:

$19 / 03 / 2020$

TEC EMPRESARIAL

VOL. 14 NO. 2, PP. 64-83 


\section{INTRODUCCIÓN}

E

l emprendimiento ha representado un pilar fundamental en el desarrollo económico y social de los países. Su importancia radica en la convergencia de ejes estratégicos del crecimiento económico, como la equidad, la innovación y el desarrollo productivo, para un óptimo accionar en el mercado (Kantis et al., 2018). Por otro lado, si bien se ha hecho notar la importancia de fomentar una cultura emprendedora en la economía, un foco de interés ha estado en la población joven. En este grupo existen ciertas aptitudes y capacidades que son fácilmente aprendidas y ha sido reconocido que estos jóvenes emprendedores propician múltiples beneficios a la economía en ámbitos de innovación, incorporación de tecnología, inserción en mercados globales, fuentes de empleo, fortalecimiento del tejido empresarial, así como una mejor distribución de la riqueza (Camacho, 2007).

Lo anterior pone de manifiesto la importancia de incentivar prácticas, entre distintos actores de la economía, que proporcionen herramientas adecuadas para potenciar el emprendimiento juvenil. La literatura ha aportado conocimiento en lo referente a ecosistemas emprendedores (Isenberg, 2010; Acs, 2014; Brown \& Mason, 2017). No obstante, el ámbito de ecosistemas emprendedores universitarios es una línea de interés que requiere mayor estudio (Morris, et al., 2017). Existen autores que han abordado factores asociados a un ecosistema emprendedor universitario, tales como el impacto de los equipos multidisciplinarios sobre la intención emprendedora (Bell, 2015), el ecosistema emprendedor digital (Sussan \& Acs, 2017), el diseño curricular, los programas co-curriculares y la asignación de recursos financieros (Morris et al., 2017; Hechavarría \& Ingram, 2018, el abordaje metodológico de los cursos de emprendimiento (Belitski \& Heron, 2017), el impacto del emprendimiento a nivel nacional (Camacho, 2007; Morris et al. 2017), la intención emprendedora (Adekiya \& Ibrahim, 2016; Nabi \& Holden, 2008), capacidades y habilidades emprendedoras (Kantis, et., 2018; Kuratko et al., 2017), entre otros tópicos.

Es por esto que abordar un modelo conceptual de ecosistema emprendedor universitario resulta de interés, particularmente, uno que permita comprender posibles

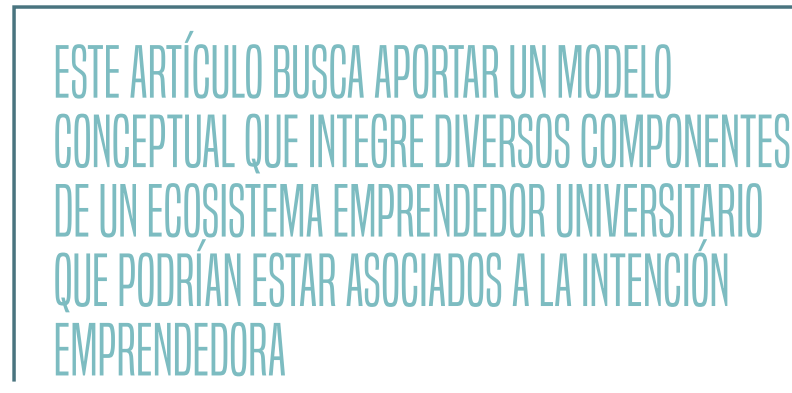

dimensiones y componentes asociados, desde un enfoque integrado. Igualmente, que se oriente a establecer el posible vínculo de sus dimensiones con la intención emprendedora de los jóvenes universitarios. Por este motivo, el presente estudio se propone buscar respuesta a la siguiente interrogante: ces posible integrar distintas dimensiones de un ecosistema emprendedor universitario que guarden relación con la intención emprendedora de los jóvenes universitarios?

Por consiguiente, este trabajo tiene por objetivo explorar el vínculo de posibles dimensiones de un ecosistema emprendedor universitario en la intención emprendedora de los jóvenes universitarios. Para ello, el estudio siguió un enfoque de investigación cualitativo, que inició con la identificación de un modelo conceptual de ecosistema emprendedor universitario a partir de una revisión de literatura especializada y reciente en esta temática, como punto de partida para ser evaluado mediante la identificación de temas y subtemas expresados en diez entrevistas a profundidad aplicadas a profesionales que laboran en unidades que tienen a cargo programas, o muy ligados a actividades, de fomento emprendedor en universidades costarricenses, estatales y privadas.

Este trabajo contribuye al ofrecer una propuesta de modelo conceptual que integre distintos componentes asociados a dimensiones de un ecosistema emprendedor universitario que podrían determinar la intención emprendedora de jóvenes universitarios. Se espera que los resultados del estudio sean de utilidad para tomadores de decisión ligados a programas e iniciativas que fomenten el emprendedurismo dentro de las instituciones de educación superior, especialmente, al considerar temas que resulten claves en la discusión de líneas de acción de sus organizaciones con miras a fortalecer y promover conductas emprendedoras en sus estudiantes. Asimismo, 
se espera que sea una base que permita ampliar la discusión y el estudio de nuevas dimensiones y temas con miras a comprender la dinámica interna de estos ecosistemas y su rol en la intención emprendedora de jóvenes universitarios.

El documento se estructura en cuatro apartados. El primero se dedica a conceptualizar aspectos relativos al emprendimiento, intención emprendedora y ecosistema emprendedor. El segundo apartado presenta aspectos metodológicos que conllevó el estudio. El tercer apartado se estructura en dos secciones, la primera de ellas muestra dimensiones o pilares que constituirían un modelo conceptual de ecosistema emprendedor universitario, los cuales surgieron de la revisión de literatura; y la segunda, presenta el resultado del análisis de los datos cualitativos surgidos de las entrevistas. Finalmente, el último apartado se dedica a sintetizar los principales hallazgos, implicaciones del estudio, limitaciones y posibles líneas futuras de investigación.

\section{REVISIÓN DE LITERATURA}

\section{EMPRENDIMIENTO}

El emprendimiento cobra un papel fundamental en el desarrollo económico y social de los países (Acs, et al., 2014). Su importancia radica en el impacto que generan las pequeñas empresas al crecimiento económico y la generación de empleo (Rideout \& Gray, 2013). En particular, el fenómeno empresarial del emprendimiento hace referencia al proceso mediante el cual se exploran, evalúan y explotan oportunidades para crear nuevos bienes y servicios (Stam, 2015). Lo anterior es materializado por el emprendedor, reconocido no solo como quien posee la visión de identificar una innovación y adaptarla de forma que se pueda comercializar en el mercado (Acs et al., 2018), sino también como quien posee la oportunidad de desarrollar múltiples habilidades, gozar de independencia, obtener beneficios financieros y contribuir al desarrollo económico de una región (Morris et al., 2017). Como resultado asumen el papel de empresarios innovadores, capaces de dar soluciones a problemas, generar empleo y riqueza (Camacho, 2007). Precisamente, el marcado surgimiento de nuevos negocios ha provocado la necesidad de comprender con mayor detenimiento el origen de dicha tendencia para potenciar los resultados (Audretsch et al., 2018).

El emprendimiento no se genera de forma espontánea y aislada, sino que responde a la interacción de diversos factores (Rangel et al., 2015) dado que las relaciones de entrada-salida son usualmente múltiples, esto es, que entradas variadas generan salidas diferentes (Audretsch et al., 2018). En consonancia con lo anterior, la literatura ha acuñado el término de ecosistemas en el ámbito empresarial, o bien, redes de sistemas y subsistemas caracterizados por la interacción dinámica en un contexto específico (Acs et al., 2017), la cooperación y las externalidades de la red de contactos (Audretsch et al., 2018). A partir de dichas propiedades, es posible que la decisión de seguir un camino emprendedor se facilite por medio de entornos de apoyo, los cuales surgen en los ecosistemas emprendedores (Morris et al., 2017).

\section{ECOSISTEMA EMPRENDEDOR}

Los ecosistemas emprendedores representan una aglomeración de agentes interconectados en un área geográfica que apoyan colectivamente la actividad empresarial (Hechavarría \& Ingram, 2018; Morris et al., 2017). Si bien es cierto, la literatura no ha proporcionado un modelo de emprendimiento estandarizado (Audretsch et al., 2018), sí se han postulado diversos factores que coadyuvan la formación de complejas estructuras socioeconómicas, denominadas ecosistemas emprendedores (Acs et al., 2017; Belitski \& Heron, 2017).

Como lo muestra la figura 1, Acs et al. (2017) presentan un marco conceptual con diversos componentes que podrían condicionar el fenómeno emprendedor. En un primer nivel se encuentran elementos y actores del macroentorno, que podrían incidir en su potencial crecimiento, como lo serían políticas de gobierno, condiciones infraestructurales, de financiamiento, educativos, entre otros. De forma global, las condiciones del marco emprendedor regulan la selección de los nuevos emprendedores y permiten conocer el grado en que las nuevas empresas resultantes pueden alcanzar su potencial de crecimiento. El primer aspecto mencionado se controla a través de condicionantes blandos, tales como las normas sociales y las preferencias culturales. 


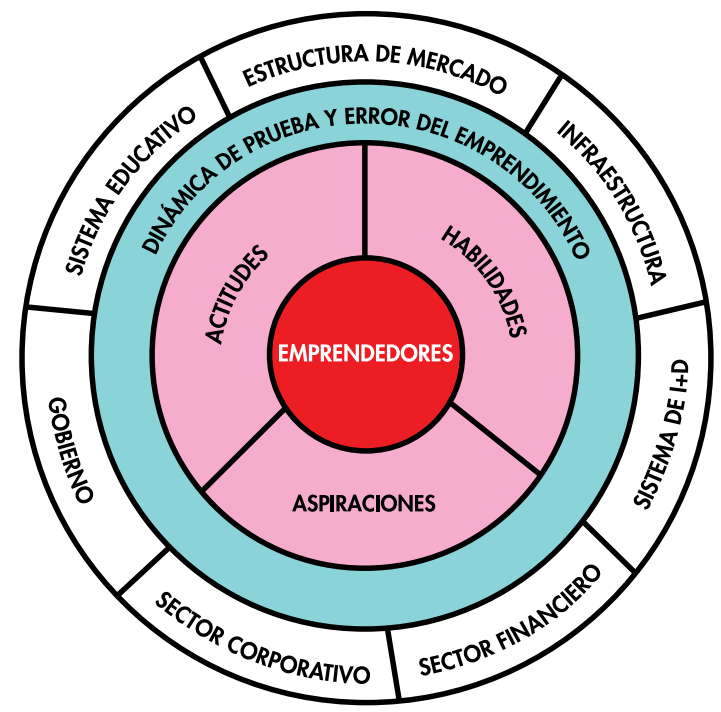

Figura 1. Condiciones del marco emprendedor Fuente: Acs, et al. (2017).

En un segundo componente, los emprendedores nacientes estimulan la dinámica de prueba y error al aventurarse en nuevos negocios para explotar oportunidades de emprendimiento con la incertidumbre de si la oportunidad es real o no. Finalmente, su esfuerzo emprendedor estaría influenciado por actitudes, aspiraciones y habilidades personales. En cuanto a las actitudes, estas pueden llegar a influir en la elección del sujeto emprendedor si prevalecen en la población. Por otra parte, a cada emprendedor naciente se le asocia con un nivel determinado de habilidades y aspiraciones emprendedoras que lo caracterizan y diferencian de los demás sujetos (Acs et al., 2017).

\section{ECOSISTEMA EMPRENDEDOR UNIVERSITARIO}

Las condiciones del marco emprendedor brindan un panorama sobre los factores que impactan el surgimiento de nuevos emprendedores (Acs et al., 2017); no obstante, la existencia de ecosistemas no es exclusiva a nivel país, pueden ser delimitados en entornos micro, dando paso a los ecosistemas nacionales, regionales, comunitarios y, más recientemente, han calado al contexto universitario (Morris et al., 2017). En particular, los ecosistemas emprendedores universitarios cobran interés como parte del modelo de la Triple Hélice, el cual posiciona a las instituciones de educación superior en el centro de las relaciones gobiernouniversidad-industria, y destaca el papel que juegan las universidades en temas de innovación, desarrollo económico (Belitski \& Heron, 2017) y en la formación de capacidades y habilidades emprendedoras (Kantis et al., 2018). Más aún, la educación se considera el eje que forma el capital humano emprendedor, concepto que hace referencia a los sujetos que cuentan con la vocación y motivación de emprender a través de un sendero de crecimiento significativo (Kantis et al., 2018).

\section{INTENCIÓN EMPRENDEDORA}

Si bien, la participación de las universidades resulta ser un aspecto crucial en el fomento del emprendimiento (Shirokova, et al., 2015), su rango de acción no debe limitarse únicamente a temas académicos - programas curriculares-, sino también en propiciar condiciones de apoyo al emprendimiento (Minola et al., 2016). La intención emprendedora de los jóvenes es un foco de interés a ser potenciado por las instituciones de educación superior, el cual refleja la predisposición de los estudiantes por participar en prácticas emprendedoras una vez finalizados sus estudios (Adekiya \& Ibrahim, 2016). A este concepto se le atribuyen tres dimensiones que la caracterizan: la actitud hacia emprender, el deseo o aspiración por emprender, y la capacidad para emprender (Ajzen, 1991; Sieger et al., 2019).

Es común que las intenciones emprendedoras de un universitario reflejen su futuro comportamiento emprendedor (Shirokova et al., 2015); no obstante, podrían presentar considerables variaciones debido a los antecedentes personales, así como los niveles y tipos de experiencias vivenciados que modulan su comportamiento (Morris et al., 2017). Como resultado, la universidad podría fomentar el desarrollo del control conductual percibido al aumentar las competencias y habilidades (Shirokova et al., 2015) a través de programas curriculares, actividades co-curriculares y la asignación de recursos financieros. Estas tres áreas podrían contrarrestar las irregularidades conductuales (Morris et al., 2017), con el fin de incentivar la intención emprendedora en los jóvenes universitarios para lanzar una empresa viable (Shirokova et al., 2015). 


\section{METODOLOǴ́A}

Para el logro del objetivo del estudio se siguió un diseño de investigación cualitativo en cinco etapas (tabla 1). La primera consistió en una revisión literaria especializada relativa a ecosistemas emprendedores universitarios, para identificar componentes que podrían estar asociados a la intención emprendedora de los jóvenes y agentes que jugarían un papel importante en el fomento en su intención emprendedora. Se siguió el procedimiento recomendado por Hakala (2011) para una revisión sistemática de literatura, que inicia con una búsqueda de artículos según palabras clave, seguido de la documentación de los papers en una hoja de cálculo MS Excel, para finalizar con la selección del contenido sustancial para la investigación (p.217). Se recurrió a revistas de alto impacto en el área de economía y negocios, tomando como referencia el factor de impacto del Journal Citation Report de Clarivitate, así como el CiteScore de Scopus. Se prestó atención particular a publicaciones análogas al emprendimiento recientes (2015 al 2019) y se utilizaron las siguientes palabras clave de búsqueda: entrepreneurial ecosystem, university ecosystem, students' entrepreneurial intentions, entrepreneurship, youth entrepreneurship y entrepreneurial ecosystem model por medio de los operadores lógicos AND y OR. Se identificaron diez revistas internacionales (tabla 2).

Una vez identificadas las revistas y analizados los artículos, se seleccionaron cuarenta y dos artículos afines a la temática, de dos de las revistas mencionadas anteriormente. De estos cuarenta y dos artículos, veintinueve permitieron generar, como producto final de esta etapa, un modelo conceptual que será descrito en el siguiente apartado, haciendo hincapié en los elementos de mayor recurrencia, o bien, contemplados en otros modelos.

En la segunda etapa, la selección de los casos de estudio respondió a dos criterios generales y cinco específicos para la unidad de análisis. En los primeros se siguió lo sugerido por Merkens (2004), que los casos transmitieran, de forma previa, una idea clara que potencialmente respondería los vacíos de información y que se obtuvieran a partir de una técnica de muestreo factible. En los específicos al perfil de universidad, se estableció que: fuese una universidad participante en el Estudio Global sobre Espíritu Emprendedor Universitario, GUESSS por sus siglas en inglés (Global University Entrepreneurship Spirit Students' Survey) o, en su defecto, que realicen esfuerzos en temas de emprendimiento; el tipo de institución -universidades privadas y universidad públicas - y años de experiencia de

Tabla 1. Etapas de la fase del estudio de campo

\begin{tabular}{|c|c|}
\hline ETAPAS & DESCRIPCIÓN \\
\hline Etapa I & Revisión de la literatura especializada. \\
\hline \multirow{2}{*}{ Etapa II } & Selección de los casos de estudio. \\
\hline & Diseño de la guía de entrevista. \\
\hline \multirow{3}{*}{ Etapa III } & Aplicación de las entrevistas. \\
\hline & Transcripción de la información. \\
\hline & Validación de los contenidos. \\
\hline Etapa IV & $\begin{array}{l}\text { Preparación para el procesamiento de datos en dos ciclos: I. Exploratorio (nube de palabras, códigos } \\
\text { provisionales, codificación abierta, libro de códigos y software NVIVO 12.5.0.) y II. Agrupamiento } \\
\text { (diagramas de afinidad). }\end{array}$ \\
\hline Etapa V & Preparación para redactar resultados. \\
\hline
\end{tabular}


programa - académico o unidad institucional-en materia de emprendimiento.

Referente a la unidad informante de cada institución de educación superior, el criterio de selección fue un profesional que, por su formación académica, tuviese alto vínculo con el emprendimiento, ya sea porque se desempeña como coordinador o responsable de un programa o unidad dedicada a actividades de emprendimiento, o bien por su interés particular en la temática dentro de la universidad. La tabla 3 muestra el perfil de universidades y entrevistados de este estudio. La guía de entrevista siguió un diseño semiestructurado de ocho preguntas abiertas, relativas a los pilares del modelo

Tabla 2. Revistas de revisión literaria

NOMBRE DE LA REVISTA

1. Journal of Business Venturing

2. Entrepreneurship Theory and Practice

3. Small Business Economics

4. Strategic Entrepreneurship Journal

5. Entrepreneurship and Regional Development

6. International Entrepreneurship and Management Journal

7. Journal of Small Business Management

8. Journal of Small Business and Enterprise Development

9. Academy of Management Journal

10. Harvard Business Review

Tabla 3. Perfil de universidad y entrevistados

\begin{tabular}{cccccc}
\hline \hline Nomenclatura & $\begin{array}{c}\text { Tipo de } \\
\text { universidad }\end{array}$ & Programa ${ }^{a}$ & Género & $\begin{array}{c}\text { Duración de } \\
\text { la entrevista }\end{array}$ & $\begin{array}{c}\text { Medio de } \\
\text { aplicación }\end{array}$ \\
\hline \hline A & Pública & Institucional & Mujer & $58,42 \mathrm{~min}$, & Presencial \\
B & Privada & Académico & Hombre & $93,10 \mathrm{~min}$, & Virtual \\
C & Privada & Institucional & Mujer & $62,75 \mathrm{~min}$, & Virtual \\
D & Privada & Institucional & Hombre & $33,19 \mathrm{~min}$, & Virtual \\
E & Privada & Institucional & Hombre & $99,12 \mathrm{~min}$, & Virtual \\
F & Privada & Académico & Hombre & $37,38 \mathrm{~min}$, & Virtual \\
G & Pública & Institucional & Mujer & $36,33 \mathrm{~min}$, & Virtual \\
H & Pública & Institucional & Mujer & $38,36 \mathrm{~min}$, & Presencial \\
I & Pública & Institucional & Hombre & $33,38 \mathrm{~min}$, & Presencial \\
J & Pública & Institucional & Mujer & $50,01 \mathrm{~min}$, & Virtual \\
\hline \hline
\end{tabular}

\footnotetext{
${ }^{a}$ Institucional = la universidad cuenta con un programa consolidado, una oficina, una incubadora o vicerrectoría dedicada al fomento del emprendimiento.

Académico = una escuela o facultad de la universidad desarrolla iniciativas de emprendimiento.
} 
de ecosistema emprendedor, para conocer su opinión en torno a la presencia de actores institucionales y fuera de la universidad; elementos de los componentes previstos y no previstos en el modelo y su posible vínculo de estos pilares con la intención emprendedora de sus estudiantes.

La tercera etapa de la metodología se dividió en tres fases: la aplicación de la entrevista, la transcripción de la información y la validación de los contenidos con la unidad informante de cada institución. En la primera de ellas, se apuntó al método de entrevista a profundidad caracterizado por la interacción verbal dentro de un proceso de acción recíproca (López \& Sandoval, 2016). De las diez entrevistas, tres se realizaron de forma presencial, con grabación en audio en los recintos de cada universidad, y las restantes siete se realizaron a través de la plataforma digital de videoconferencia remota Zoom, con grabación de video en la nube. En la segunda fase se procedió a transcribir, "palabra por palabra", cada una de las entrevistas en un archivo Word. Se procedió a una segunda sesión con cada entrevistado para revisar, conjuntamente, un resumen y esquema gráfico de los principales aspectos que el entrevistado manifestó en cada una de las preguntas, verificar que reflejara todo lo que expresó, corregir algún aspecto no comprendido a la hora de transcribir la entrevista o agregar algún aspecto adicional no considerado en la primera entrevista. Con ello, se procedió a ajustar el texto transcrito en lo correspondiente. En promedio, las dos visitas de entrevistas tuvieron una duración de 54 minutos.

Una cuarta etapa se centró en la preparación de los datos textuales para su procesamiento en dos ciclos: exploratorio para generar códigos y el de patrones de códigos o agrupamiento, para identificar categorías o temas (Miles, et al., 2014; Saldaña, 2013). Los datos textuales se procesaron en el software NVIVO, 12.5.0, iniciando con una exploración de textos mediante el uso de nubes y árboles de palabras. Lo anterior con el propósito de identificar contenidos en torno a palabras de mayor frecuencia de mención entre los entrevistados, como una fuente provisional de identificación de códigos iniciales. A lo anterior se sumó una lista de otros códigos provisionales que resultaron de la revisión de la literatura para cada uno de los pilares del modelo conceptual. Con ello se fue generando un libro de códigos, o un compendio estructurado de códigos, y la forma en que estos se relacionan (Guest et al., 2011). Se procedió a realizar una codificación abierta y una subcodificación de códigos de segundo orden (Miles et al., 2014). Seguidamente, se procedió a la fase de patrones de códigos para identificar categorías o temas, analizando la recurrencia de los temas que permitiera identificar categorías y subcategorías temáticas implícitas en el contenido de los datos, para contrastar y analizar el esquema teórico (Guest et al., 2011). Una vez codificados los datos textuales, se tomó cada uno de ellos para ser agrupados según afinidad, por lo cual, se recurrió a la técnica del Diagrama de Afinidad, según lo sugerido por Asaka \& Ozeki (1988). Finalmente, la última etapa recurrió al enfoque descriptivo narrativo, sugerido por Miles et al. (2014), para presentar los resultados del análisis de los temas.

\section{ANǴLISIS Y DISCUSIÓN}

\section{PILARES DE UN ECOSISTEMA EMPRENDEDOR UNIVERSITARIO: RESULTADO DE LA REVISIÓN DE UTERATURA}

Un primer pilar de un ecosistema universitario vendría a ser los programas curriculares. Conviene enfatizar que la educación emprendedora es el desarrollo de actitudes, comportamientos y capacidades aplicables durante la carrera de emprendedor (Bell, 2015). Asimismo, se ha reconocido que impacta, de forma positiva, al capital humano, sus creencias, así como su habilidad para aprovechar oportunidades (Morris et al., 2017; Shirokova et al., 2015). Se ha reconocido que, traducido a los programas curriculares, estos favorecen el panorama para los jóvenes emprendedores mediante la transmisión de conocimientos; no obstante, el simple hecho de ofrecer una currícula cargada de cursos y programas emprendedores no garantizaría el éxito de los individuos (Morris et al., 2017). Por ello, la metodología de aprendizaje y el formato en que se imparten los cursos pueden ser determinantes en la intención emprendedora (Morris et al., 2017; Rangel et al., 2015).

En este sentido, ha sido sujeto de reflexión si los espacios curriculares, en términos de número de horas semestrales, la obligatoriedad de la evaluación numérica y los criterios de homogeneización, permiten una adecuada formación para los emprendedores nacientes, 
o representan solamente conocimientos formales que conducen al sujeto a una situación pasiva frente a su propio proceso de aprendizaje (Rangel et al., 2015). Por ejemplo, Bell (2015) señala que las metodologías basadas en el aprendizaje activo y en el experimental incrementan la motivación de la comunidad estudiantil. El aprendizaje activo requiere mayores habilidades de análisis, síntesis y evaluación; mientras que el experimental representa una forma participativa de aprender a través del esfuerzo mental para sintetizar y extraer información en un entorno cambiante (Bell, 2015). El trabajo de equipos multidisciplinarios representaría no solo un potenciador en ambas metodologías sino también un componente trascendente en el ecosistema emprendedor universitario (Morris et al., 2017).

Un segundo pilar lo conformarían las actividades cocurriculares, esto es, aquellos esfuerzos de aprendizaje que se dan fuera de los salones de clase. Por un lado, estos programas simulan experiencias emprendedoras, permitiendo a los estudiantes poner en práctica conocimientos adquiridos en el aula y, por otro lado, les ofrece la posibilidad de desarrollar una red de contactos como resultado de la relación con entes de diversos sectores que pueden ser provechosos en el futuro (Morris et al., 2017).

De igual modo, se ha dicho que las actividades cocurriculares incentivan habilidades emprendedoras en los estudiantes, entre las cuales destacan la toma de riesgos, la proactividad y la innovación (Bell, 2015). Puntualmente, la toma de riesgos es una característica importante debido a que los emprendedores suelen presentar mayor disposición ante situaciones de riesgo que las demás personas, y la proactividad se centra en lograr objetivos y hacer que las cosas sucedan (Bell, 2015). La innovación es considerada como la fuerza que guía los procesos de emprendimiento, permitiendo el surgimiento y ejecución de nuevas ideas, procesos, productos o servicios (Kuratko et al., 2017).

Los recursos financieros desempeñan un papel fundamental en el éxito de una idea de negocios al materializar las ideas de los emprendedores, representando un tercer pilar en los ecosistemas emprendedores. Aplicado al contexto universitario, la dotación de recursos financieros podría verse reflejada en una plataforma de facilidades que ofrece la institución para que los estudiantes puedan explotar su intención emprendedora. Morris et al. (2017) señalan que los recursos financieros que destinan las universidades al emprendimiento se verían reflejados en los cursos de emprendimiento, la oferta de grados académicos, el compromiso de ex alumnos emprendedores de compartir sus experiencias vividas y aprendizajes adquiridos con los nuevos emprendedores, incubadoras de ideas donde destacan los servicios de desarrollo de prototipos, el capital semilla otorgado a los proyectos como medio de financiamiento de la idea de negocios, la transmisión de tecnologías, la investigación, entre otros.

Un cuarto pilar podría ubicarse dentro del ámbito del compromiso estratégico de la universidad, que impacte la intención emprendedora de los jóvenes por medio del compromiso de liderazgo de todos los miembros del ecosistema, el compromiso de innovación constante y el compromiso de la universidad sostenido a través del tiempo (Rice et al., 2014). Se debe tomar en consideración que el emprendimiento no necesariamente está relacionado con la creación de una organización o la generación de un beneficio económico (Rangel et al., 2015). Cada universidad puede tener diferentes objetivos y estrategias relacionadas al tema de emprendimiento, sin embargo, se ha reconocido que la claridad en la línea estratégica incide en el emprendimiento universitario de los jóvenes (Wright et al., 2017).

Por su parte, un quinto pilar sería la participación de la universidad en redes globales académicas y de emprendimiento. Estas podrían brindar, a los estudiantes, oportunidades de desarrollo académico a una escala global, propiciar ciudadanos dinámicos, modular el pensamiento abierto en los jóvenes y educar a los estudiantes para que cuenten con capacidades y habilidades necesarias para desenvolverse en un entorno local, así como adaptarse a un ambiente y una cultura extranjera diferente. En adición, los estudiantes que se exponen a la participación en redes globales contarían con mejor información sobre los mercados internacionales, una variedad de recursos, nuevas y mejores prácticas, la posibilidad de desarrollar proyectos con un menor riesgo asociado y, finalmente, se relacionarían con una red de contactos (Minola et al., 2016).

Por otra parte, la infraestructura organizacional se conformaría en un sexto pilar dentro del ecosistema 
emprendedor universitario, que podría permitir consolidar en doble vía, el compromiso de la comunidad emprendedora con los agentes internos a la universidad que se encargan de la formulación de políticas que impactan el emprendimiento en los estudiantes (Picado et al., 2015). De esta forma, la estructura propicia espacios de interacción con la red de contactos local y de puestos clave a lo interno de la institución, pudiendo lograr un acercamiento que se traduciría en un derrame de conocimiento, la transmisión de iniciativas de investigación y una mayor comprensión de los intereses de cada una de las partes (Belitski \& Heron, 2017).

Por último, cabe señalar que, al igual que la mayoría de las actividades e interacciones humanas, el emprendimiento se encuentra estrechamente relacionado con la tecnología (Acs et al., 2017). Debido a esto, el modelo de ecosistema emprendedor se podría proyectar en la era digital. Recientemente, se plantea la idea de que un ecosistema emprendedor, inmerso en la era digital, es un sistema auto-organizable, escalable y sostenible, compuesto por entidades digitales heterogéneas que buscan aumentar la utilidad del sistema y obtener beneficios, así como promover el intercambio de información, la cooperación interna e interoperativa, y la innovación. A partir de lo anterior, la intención emprendedora de los jóvenes podría estar influenciada por las nuevas tendencias, plataformas y descubrimientos del área digital (Sussan \& Acs, 2017).

La figura 2 permite sintetizar la propuesta teórica de posibles pilares y componentes asociados a un ecosistema

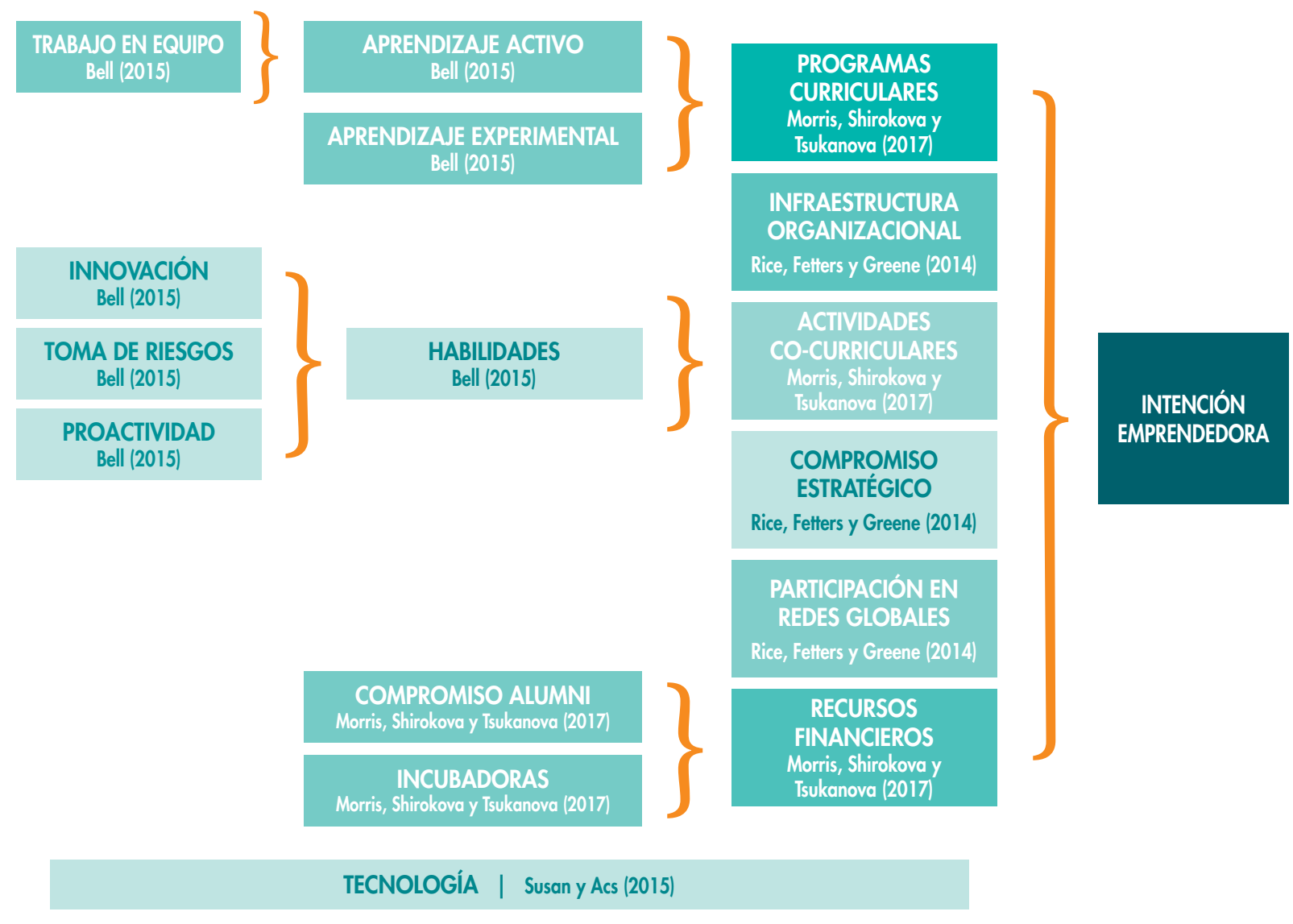

Figura 2. Síntesis del modelo

Fuente: Elaboración propia con información de Morris et al. (2017), Rice et al. (2014), Susan \& Acs (2017), y Bell (2015). 
emprendedor universitario que podría determinar la intención emprendedora de los jóvenes universitarios. Esta propuesta sugiere la dimensión de la tecnología como un posible eje transversal del modelo de ecosistema emprendedor universitario.

\section{CONCEPCIÓN DEL MODELO Y SU POSIBLE VÍNCULO CON LA INTENCIÓN EMPRENDEDORA: RESULTADO DEL ANÁLISIS DE DATOS CUALTATIVOS}

El proceso de codificación de textos generó 148 expresiones verbales emanadas por los entrevistados. En la figura 3 se puede observar que cerca de seis de cada diez datos textuales se concentraron en subtemas que giraron en torno a temas curriculares y co-curricuales. En este apartado se sintetizarán los contenidos de las expresiones verbales de los principales subtemas en cada uno de los pilares y el vínculo percibido por los entrevistados con la intención emprendedora de los jóvenes universitarios. Para ello, se siguió el enfoque de descripción narrativa sugerido por Miles et al. (2014). En el apéndice 1, el lector puede observar los subtemas y la frecuencia de textos de cada uno de ellos, en relación con el total de su pilar y el total de citas.

\section{PROGRAMAS CURRICULARES}

De las diez universidades, nueve imparten cursos de emprendimiento para su comunidad estudiantil. De ellas, cuatro ofertan el curso transversal a todas las carreras cuyas clases combinan estudiantes de diversas áreas, lo cual "promueve el trabajo multidisciplinario" (entrevistado A) y "les enseña a trabajar juntos y aprender de las diferencias" (entrevistado C). En este pilar surgieron categorías temáticas con contenidos afines a cuatro grandes subtemas; a saber, metodología (17 de 44 citas), perfil docente (14 de 44), beneficios (7 de 44) y predisposición emprendedora (6 de 44). Al ser consultados si el diseño de los programas curriculares influye en la intención emprendedora de los jóvenes universitarios en lo relativo a beneficios que le aporta en su experiencia emprendedora, algunos entrevistados

\section{Distribución de los componentes del modelo de ecosistema emprendedor según la cantidad de fragmentos de texto en términos absolutos para el 2019.}

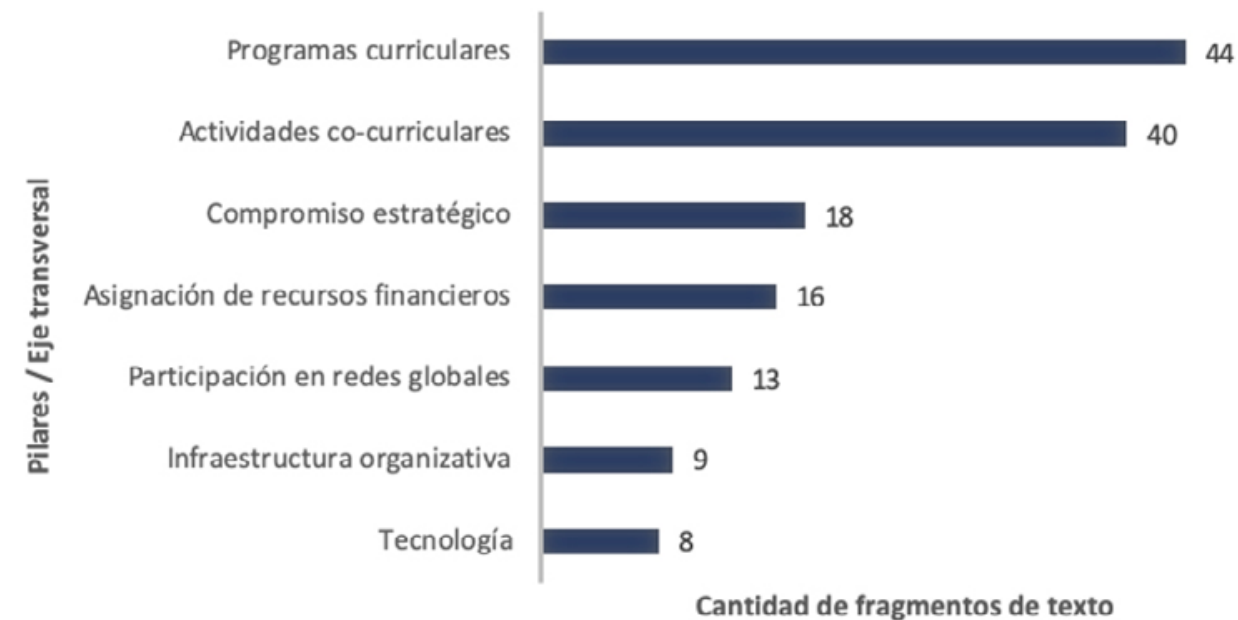

Figura 3. Distribución de los componentes del modelo según cantidad de fragmentos de texto Fuente: Elaboración propia 
mencionaron repetidamente la palabra "despertar" (entrevistados A, C y J) y "ayudar" (entrevistado H) cuyo texto hacía referencia a la forma en que los cursos de emprendimiento despiertan interés en los jóvenes. A pesar de que el diseño curricular no es el único factor que influye en la intención emprendedora de los universitarios, afirman que estos contribuyen sustancialmente al descubrimiento de las habilidades emprendedoras y la consolidación de un pensamiento crítico: "la universidad lo lleva por un proceso donde sea él quien busca la solución" (entrevistado C). En el subtema de predisposición emprendedora, cinco entrevistados (A, C, D, E, J) fueron próximos en expresar que el descubrimiento de la intención emprendedora se asocia a aquellos estudiantes que, previamente, experimentaron curiosidad en materia de emprendimiento: "Yo he visto que las personas realmente se desarrollan cuando quieren algo, y ċcuándo quieren?, cuando usted tiene ahí esa chispita" (entrevistado A).

En lo referente al pilar de metodología de los programas curriculares, se hace hincapié en el aprendizaje experimental y activo como un elemento positivo a la intención emprendedora. Por ejemplo, cinco entrevistados comentaron que sus programas han adaptado una metodología de "aprender haciendo" (8 fragmentos de texto de los entrevistados A, D, E, F y G). Además, sugirieron técnicas activas, lúdicas y constructivistas (7 fragmentos de los entrevistados A, D, E, F, G y J) para abordar la temática de emprendimiento porque: "permite al estudiante ir desarrollando toda su intención emprendedora y empresarial, en conjunto con el cliente o sector al que va orientado" (entrevistado G).

En este pilar surge un elemento no abordado en el modelo conceptual del ecosistema emprendedor universitario, el rol del profesor como estímulo de la intención emprendedora de los estudiantes. Un elemento análogo en tres de los entrevistados (B, C, J) es que, a pesar de la dificultad de describir el perfil de un profesor de emprendimiento, reconocen su experiencia, un mindset de emprendimiento, su intencionalidad por potenciar en sus estudiantes la propósito emprendedor: "Yo creo que es el profesor, porque yo puedo hablarte y darte una charla y hacerte un speech motivador, puedo usar el caso, puedo usar la simulación, puedo usar cualquier cosa que me des, yo la convierto en un mensaje emprendedor. Si yo no tengo el mindset, si yo no estoy impulsando con intencionalidad

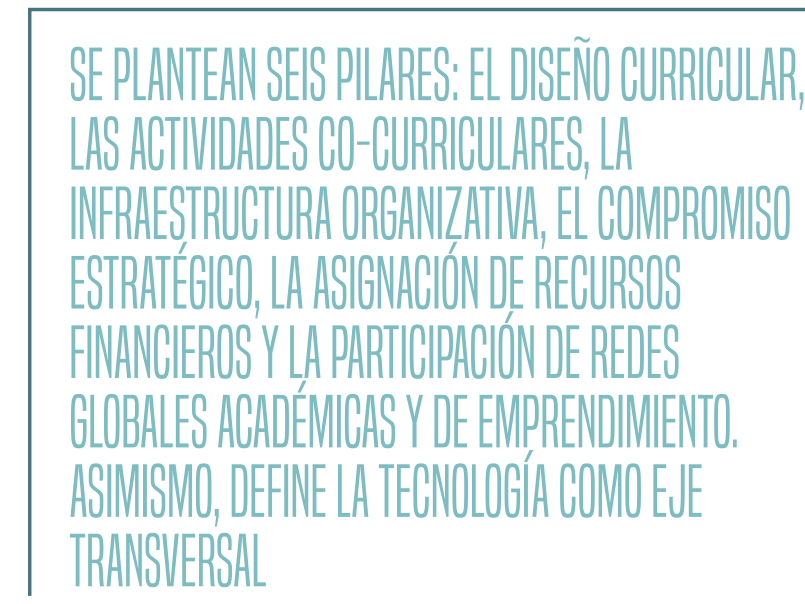

el emprendimiento, me puedes poner la metodología que ha sido exitosa en cualquier parte que yo no la voy a explotar" (entrevistado B); e igual de importante es su perfil investigador.

Finalmente, algunos entrevistados abordaron la categoría temática de la obligatoriedad de los cursos (3 fragmentos de texto de entrevistados A, B y C), donde se polarizan las opiniones de los entrevistados. Unos apuntaron a que la obligatoriedad de los cursos no despierta la intención emprendedora y responde únicamente a una responsabilidad curricular; mientras que otro señala que este formato garantiza la transversalidad a todas las carreras y, por ende, el trabajo en equipos multidisciplinarios. De forma complementaria, el entrevistado $\mathrm{C}$ comparte que: "cuando se nos piden decisiones importantes siendo tan jóvenes, muchas veces no consideramos que tenemos la actitud, el deseo o la capacidad, y por eso se hacen obligatorios los cursos".

$\mathrm{Al}$ contrastar la información obtenida del primer pilar con los aportes realizados por diferentes autores, se puede observar que la discusión sobre la obligatoriedad de los cursos ya se ha generado anteriormente. Rangel et al. (2015) establecen que es pertinente "reflexionar si los espacios de una cátedra, en términos de horas semestrales, obligatoriedad de la evaluación numérica y criterios de homogenización, permiten una adecuada formación o brindan conocimientos formales que llevan al estudiante a una situación pasiva de aprendizaje" (p. 123). 


\section{ACTIVIDADES CO-CURRICULARES}

Este pilar generó cuatro subtemas, a saber, iniciativas co-curriculares (17 de 40), su impacto (14 de 40), el origen de estas iniciativas ( 8 de 40) y el papel de la universidad (1 de 40). Los talleres, las ferias de ideas de negocios y los retos son las actividades co-curriculares más mencionadas por los entrevistados como parte de su oferta co-curricular. En general, los contenidos de los textos de seis de los diez entrevistados son próximos a considerar que este tipo de actividades influyen de manera complementaria en la intención emprendedora porque requieren un esfuerzo adicional y, por ende, captan a los jóvenes con predisposición positiva hacia el emprendimiento. Acorde con lo anterior, si bien este tipo de actividades busca impactar a toda la comunidad, se debe visualizar "como un embudo donde existe una gran cantidad de personas que entran y pasan por diferentes actividades, pero al final pocos se comprometen y realmente crean empresa" (entrevistado A). Lo anterior se evidencia en expresiones características como: "entre un $5 \%$ y un $10 \%$ de los proyectos que nosotros tenemos tiene esa relación, o sea, provienen de algunas de las actividades que la universidad organizó" (entrevistado I), o bien que: "el 90\% de los que tenemos en incubación han pasado por alguna actividad de fomento emprendedor" (entrevistado A).

De forma complementaria, Morris et al. (2017) mencionan que las actividades co-curriculares les brindan a los estudiantes la posibilidad de conectar e interactuar con expertos de diferentes áreas, es decir, vincularse con el medio en el que se desenvuelven. Aunado a lo anterior, los autores explican que las actividades que se desarrollan fuera de la clase apoyan la confianza en sí mismos, lo cual empodera a los estudiantes sobre sus capacidades personales.

\section{COMPROMISO ESTRATÉGICO}

Tres subtemas destacados en este pilar fueron la cultura emprendedora universitaria (6 de 18), el impacto del compromiso estratégico (7 de 18) y la caracterización del compromiso (5 de 18). Al analizar la influencia del compromiso estratégico de la universidad, los resultados indican que este puede llegar a influir en la intención emprendedora de los jóvenes universitarios puesto que estimula a las instancias universitarias a involucrarse en temas de emprendimiento estudiantil: "Desde la óptica de los estudiantes, el tema del compromiso estratégico, si una universidad no está alineada, si los agentes que promueven el emprendimiento están desligados del apoyo estratégico que tengan que dar las altas autoridades, creo que poco se lograría" (entrevistado J).

$\mathrm{Al}$ abordar el tema del compromiso estratégico, algunas universidades vincularon este pilar con el concepto de cultura emprendedora (6 fragmentos de texto de las entrevistas A, B, D e I), para conectar con los estudiantes y potenciar su intención emprendedora: "si vos tirás a ese Comité de Extensión el lineamiento, vamos a fomentar el emprendimiento dentro de la universidad y propongamos actividades conjuntas a nivel universidad, se van a comenzar a realizar actividades que vayan creando la cultura o la mentalidad de que la universidad va a estimular en sus estudiantes el espíritu emprendedor" (entrevistado B).

Rice et al. (2014) mencionan que el compromiso estratégico de las universidades se propicia a través del liderazgo de todos los miembros del ecosistema, lo cual se relaciona con las perspectivas de los entrevistados, donde establecen que cuando existe un lineamiento claramente establecido, el accionar institucional en materia de emprendimiento se beneficia y fluye con mayor facilidad.

\section{ASIGNACIÓN DE RECURSOS FINANCIEROS}

Los datos textuales en este pilar se decantaron en dos grandes subtemas, apoyo indirecto de los recursos (12 de 16) y apoyo directo (4 de 16). Se denota una polarización de puntos de vista en dos vertientes: una mayoría se decantan por argumentar que las universidades no deben asignar recursos financieros a los proyectos emprendedores, puesto que no les compete el tema. Lo anterior se debe fundamentalmente a dos motivos, primero, que el emprendedor debe mostrar proactividad en la búsqueda de recursos y no solamente esperar los fondos. Segundo, si el ecosistema nacional se encuentra debidamente consolidado, se parte de la premisa que este posee los agentes para que los emprendedores puedan recurrir a fuentes de financiamiento. Debido a esto, la universidad se convierte en una plataforma de acompañamiento y asesoría para los jóvenes, así como un administrador y canalizador de los recursos económicos de los proyectos, sin tener que destinar montos de dinero 
específicos a ideas de negocios: "Pienso que la universidad tiene que dar recursos en conocimiento, acompañamiento, infraestructura, apoyo (...) porque del recurso financiero se tendrá que empeñar el emprendedor, que me parece que es parte de su iniciativa" (entrevistado J).

Por otra parte, tres de los diez entrevistados son del criterio de la necesidad de un apoyo directo de la universidad al financiamiento de proyectos emprendedores, principalmente para la validación de la idea de negocios: "Cuando los chicos desarrollan propuestas fuertes y robustas, y se topan con que la idea quedó ahí en el papel, ganaron el concurso y todo, pero no se puede materializar, es un desacelerador porque la universidad debería estar en la capacidad de generar un capital no reembolsable" (entrevistado E).

Sobre la posición de los entrevistados acerca de la plataforma de acompañamiento al emprendedor que le permite conectarse con actores clave del ecosistema externo, Rangel et al. (2015) fortalecen la idea al mencionar que "la universidad es un agente de transferencia de conocimiento y de tecnología donde la base de este proceso son la innovación y el emprendimiento, teniendo siempre como fin último el compromiso social con el entorno en el que actúa e influye" (p. 125).

\section{PARTICIPACIÓN EN REDES GLOBALES ACADÉMICAS Y DE EMPRENDIMIENTO}

El principal subtema en este perfil correspondió al impacto de las redes (12 de 13). Una mayoría de entrevistados considera que este pilar influye en la intención emprendedora de los jóvenes ante la oportunidad de tener contacto, tanto profesores como estudiantes, con prácticas y experiencias en otras universidades en emprendedurismo, lo cual representa una posibilidad para adaptar conocimiento y mantenerse actualizado: "En síntesis, de la participación de la universidad en redes globales académicas y emprendimiento, favorece adquirir y conocer nuevas y mejores prácticas, y la parte de networking" (entrevistado D); "puede aportar respecto a nuevas tendencias de emprendimiento, nuevas maneras de apoyar a los emprendedores" (entrevistado $\mathrm{H}$ ).

En adición, según los entrevistados, dicho pilar se relaciona con la cultura emprendedora, ya que las redes globales generan un ambiente de emprendimiento que incentiva, en los estudiantes, la intención emprendedora de forma directa e indirecta. Los jóvenes vivencian experiencias que les hacen conocer nuevas prácticas con realidades distintas a su vida académica, pero también reciben un derrame de conocimiento indirecto cuando sus profesores participan de actividades internacionales e implementan las técnicas o comparten sus anécdotas de las redes: "A nivel competitivo creo que es muy útil, además de que eso también te ayuda a ir descremando las tendencias mundiales hacia dónde va la gente pensando" (entrevistado E).

\section{INFRAESTRUCTURA ORGANIZATIVA}

En este pilar, el subtema dominante lo representó el impacto de la infraestructura organizacional (7 de 9). En dos de diez entrevistados se coincidió en que el carácter reglamentista, estructurado y rígido (2 fragmentos de texto de los entrevistados B e I) se convierte en un obstaculizador del accionar de las instituciones de educación superior, restando potencial efecto a la intención emprendedora de los jóvenes universitarios: "la idea del fracaso es algo que tiene que existir, pero eso implica riesgos, y la universidad en su estructura es muy minimizadora de riesgos porque solo quiere ir a la segura, usualmente en términos de rentabilidad" (entrevistado B).

En contraposición, tres de los diez entrevistados sostienen que la infraestructura organizativa es determinante en la intención emprendedora de los estudiantes ( 5 fragmentos de texto en los entrevistados C, D y H): "El hecho de que no tengamos una estructura tan compleja jerárquicamente, diría que es una cosa que influye favorablemente" (entrevistado C). Cabe mencionar que los restantes cinco entrevistados abordan el pilar de infraestructura organizativa desde una posición muy neutral y se enfocan en la descripción de los programas de emprendimiento.

$\mathrm{Al}$ analizar el fragmento de texto mencionado en la entrevista B sobre la existencia de riesgos que pueden conducir al fracaso, se puede identificar una similitud con la afirmación realizada por Rangel et al. (2015), donde establecen que "la educación en emprendimiento debe permitir los errores como posibilidades de aprendizaje" (p. 123), haciendo referencia a la importancia de una estructura que no limite el riesgo entre los estudiantes. 
Adicionalmente, los autores explican que es importante una "infraestructura que favorezca la construcción de puentes entre el Gobierno, las universidades y las organizaciones de manera transdisciplinaria” (p.118).

\section{TECNOLOGÍA}

Dos subtemas se decantaron en este pilar, puntos de vistas positivos de la viabilidad que brinda la tecnología al emprendimiento (6 de 8) y sentido de prudencia ante la tecnología (2 de 8). En general, se considera que la tecnología tiene un carácter de transversalidad, un facilitador para la intención emprendedora: "hace más fácil todo, desde conseguir información y ver qué se está haciendo en otro lado, hasta conectarse con otras personas, ver ejemplos de cómo otras personas están haciendo negocios usando esas plataformas" (entrevistado D).

A pesar de ello, dos de los diez entrevistados consideran que la tecnología influye, pero no es determinante en la intención emprendedora. Sus opiniones se enfocan en aclarar que la tecnología abre un espectro de oportunidades de emprendimiento y concibe la idea del emprendimiento con mayor simplicidad, sin embargo, muchas veces idealiza el emprendimiento, al no sustentarlo en una idea de negocios clara y estructurada: "estamos en un cambio tecnológico que, para efectos prácticos a nivel de emprendimiento, y perdón lo que voy a decir pero es que así lo pienso, hace parecer más fácil el emprender porque hay muchas ideas que pueden surgir de las nuevas tecnologías (...), pinta un cuadro macro de que va a ser relativamente sencillo, entonces, lo que ahí habría que tener es cuidado" (entrevistado A).

En lo relativo a esta percepción de que la tecnología abre nuevas dimensiones de emprendimiento que los emprendedores pueden aprovechar para generar ideas de negocios, Sussan y Acs (2017) explican que algunos de los usuarios de la tecnología desarrollan nuevos productos y servicios como resultado de una interacción intensa con la comunidad y se convierten en emprendedores. Visualizar la tecnología como un eje transversal en el modelo de ecosistema emprendedor universitario estaría respaldado con la propuesta de Sussan y Acs (2017), quienes plasman los ecosistemas digitales fuera de los ecosistemas emprendedores, suponiendo una interacción entre ellos (figura 4).

A manera de síntesis, la figura 5 busca sistematizar gráficamente los resultados obtenidos en las entrevistas realizadas, según las siete dimensiones del modelo de ecosistema emprendedor universitario.

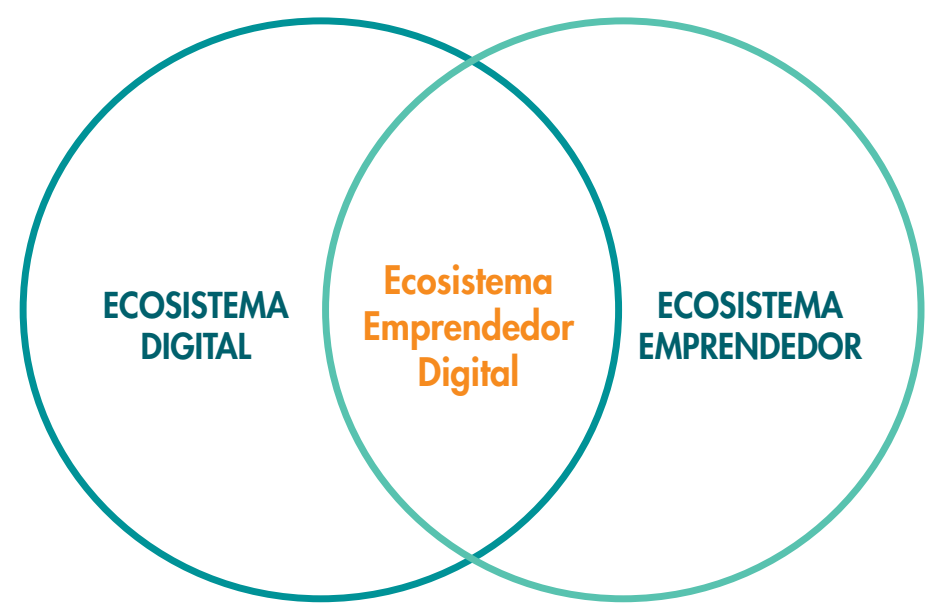

Figura 4. Interacción entre ecosistemas

Fuente: Sussan, \& Acs, (2017). 
Pilares/Eje Transversal

Programas Curriculares

Actividades Co-Curriculares

Compromiso Estratégico

Recursos Financieros

Infraestructura Organizacional

\section{Principales hallazgos de las entrevistas}

- Metodología: aprendizaje experimental acompañado de técnicas activas, lúdicas y constructivistas

- Perfil docente: el rol del profesor se convierte en un estímulo de la intención emprendedora.

- Beneficios: despiertan interés en los jóvenes, contribuyen al descubrimiento de las habilidades emprendedores y la consolidación de un pensamiento crítico.

- Predisposición emprendedora: asociada a los estudiantes que, previamente, experimentaron curiosidad en materia de emprendimiento.

- Impacto de las iniciativas co-curriculares: influyen de manera complementaria porque implican un esfuerzo adicional y por ende captan jóvenes con predisposición positiva hacia el emprendimiento.

- Origen: son el punto de partida de muchos proyectos emprendedores.

- Cultura emprendedora: permite conectar con los estudiantes y potenciar su intención emprendedora.

- Impacto del compromiso estratégico: estimula a las instancias universitarias a involucrarse en temas de emprendiento estudiantil.

- Apoyo directo: apoyo directo de la universidad, principalmente para la validación de la idea.

- Apoyo indirecto: la universidad se convierte en una plataforma de acompañamiento y asesoría para los jóvenes, así como un administrador y canalizador de los recursos económicos.

- Impacto de las redes: permite el contacto con otras universidades e instituciones, lo cual representa una posibilidad para adaptar conocimiento y mantenerse actualizarse.

- Cultura emprendedora: generan un ambiente que incentiva la intención emprendedora.

- Impacto de la infraestructura: obstaculiza al ser reglamentista, estructurada y rígida, pero otras universidades consideran que es determinante en la intención emprendedora de los jóvenes.

- Viabilidad: tiene un caracter de transversalidad, un facilitador para la intención emprendedora.

Tecnología

- Prudencia: influye, pero no es determinanteen la intención emprendedora. Concibe la idea de mayor simplicidad, sin embargo, muchas veces idealiza el emprendimiento, al no sustentarlo en una idea de negocios clara y estructurada.

Figura 5. Principales hallazgos de las entrevistas según los pilares/eje transversal del modelo de ecosistema emprendedor universitario 


\section{CONCLUSIONES}

Este trabajo tuvo como objetivo explorar el vínculo de posibles dimensiones de un ecosistema emprendedor universitario en la intención emprendedora de jóvenes universitarios. Bajo un enfoque de investigación cualitativa, en cinco etapas, a partir de una revisión reciente de literatura, se propuso un modelo conceptual de ecosistema emprendedor universitario a ser corroborado mediante entrevistas en profundidad con expertos. Dicho modelo se compone de seis pilares y un eje transversal: programas curriculares, actividades co-curriculares, compromiso estratégico, asignación de recursos financieros, participación en redes globales, infraestructura organizativa y tecnología.

En lo referente a la interrogante planteada al inicio de la investigación, ces posible integrar distintas dimensiones de un ecosistema emprendedor universitario que guarden relación con la intención emprendedora de los jóvenes universitarios? El presente estudio parece confirmar la presencia de diversos factores del ecosistema universitario asociados a la intención emprendedora de los estudiantes, que pueden ser englobados en un modelo de ecosistema emprendedor. Dado el alcance exploratorio del estudio, no es posible validar las dimensiones del modelo ni su respectivo impacto sobre la intención emprendedora.

El análisis de los datos textuales ha permitido destacar tres aspectos dentro del contexto del diseño curricular vinculados con la intención emprendedora: la metodología de aprendizaje, el perfil actitudinal del profesor y la predisposición emprendedora del joven universitario. Referente a la metodología, cursos de emprendimiento estructurados bajo una línea experimental, vivencial y activa, para que los estudiantes desarrollen habilidades y capacidades emprendedoras como respuesta a un estímulo real del medio, son valorados positivamente. Asimismo, una disposición y actitud intencional del docente por promover el espíritu en los estudiantes y motivarlos a emprender. Por otra parte, los estudiantes con predisposición emprendedora positiva se convierten en potencial foco de interés de los programas académicos que buscan impulsar la intención emprendedora.

Las actividades co-curriculares pueden ser vistas como un tamiz afín, con la predisposición hacia el emprendimiento. Por otra parte, el compromiso estratégico definido por los centros educativos superiores que permita alinear los esfuerzos departamentales hacia el emprendimiento y conformar una cultura emprendedora en colaboradores y estudiantes puede contribuir en la intención emprendedora. De igual manera, la participación en redes globales puede representar una vitrina para aportar nuevas prácticas y experiencias que estimulen la intención emprendedora.

Finalmente, el que las universidades se encuentren circunscritas en un ecosistema nacional y se articulen con otros agentes para un aprovechamiento de recursos financieros y de relaciones sería un factor por considerar en la intención emprendedora. Consecuentemente, deben apuntar a estructuras con muros permeables, en el sentido que se genere valor en doble vía, es decir, hacia afuera y hacia adentro de las universidades, lo que podría ser un facilitador de la interacción entre ambos ecosistemas (nacional y universitario).

\section{IMPLICACIONES, LIMITACIONES Y FUTURAS LINEAS DE INUESTIGACIÓN}

Las implicaciones teóricas del estudio giran en torno al modelo de ecosistema emprendedor plasmado en seis pilares y un eje transversal. La literatura científica en el tema de ecosistemas emprendedores ha crecido, y dentro de ella un área que ha estado tomando impulso ha sido el de ecosistema universitario. Este artículo aporta al hacer un intento de consolidar lo más importante de esa creciente literatura y tratar de confirmarlo con el criterio de expertos, practitioners, en universidades donde se fomenta activamente la intención emprendedora de los estudiantes. Desde la perspectiva práctica se ponen sobre la mesa algunos tópicos para analizar y discutir, como la asignación de recursos financieros, la obligatoriedad de los cursos y el compromiso estratégico institucional.

En relación con las limitaciones del proyecto, cabe señalar que, debido a que el enfoque del estudio es cualitativo y exploratorio, no es posible realizar inferencias sobre la temática de interés. Además, la revisión de la literatura, al ser exploratoria, incluyó tan solo diez revistas científicas de alto impacto. Finalmente, la convalidación del modelo se realiza únicamente en 
centros de educación superior en Costa Rica, por lo que pueden existir elementos idiosincráticos al entorno costarricense que no necesariamente se produzcan en otros contextos. Por otro lado, la confirmación de los pilares y su posible vínculo con la intención emprendedora de los jóvenes universitarios se ha realizado, únicamente, tomando la perspectiva y visión de los encargados de promover el emprendedurismo dentro de la universidad, o lo que hemos denominado como practitioners, quedando pendiente la perspectiva del joven universitario emprendedor.

Lo anterior lleva a proponer como posibles futuras líneas de investigación, profundizar en el análisis de las dimensiones del modelo propuesto mediante la comparación entre universidades de diferentes países. Igualmente, ahondar en los pilares del modelo desde la óptica de jóvenes universitarios que muestran diversos grados de intención emprendedora. Por su parte, se podría realizar estudios que permitan comprender el papel y rasgos del profesor en los cursos de emprendimiento que influyen en la intención emprendedora. Además, se convierte en un foco de interés el rol de las universidades en la asignación de recursos financieros. Finalmente, podría valorarse acometer un estudio más amplio, desde un enfoque cuantitativo, que indague el grado de influencia que ejercen los componentes del ecosistema emprendedor sobre la intención emprendedora.

\section{APÉNDICE}

Apéndice 1. Diagrama de afinidad de temas y subtemas asociados al ecosistema emprendedor universitario, a partir del agrupamiento próximo de los 148 datos textuales de las entrevistas.

\section{ECOSISTEMA EMPRENDEDOR UNIVERSITARIO 148}
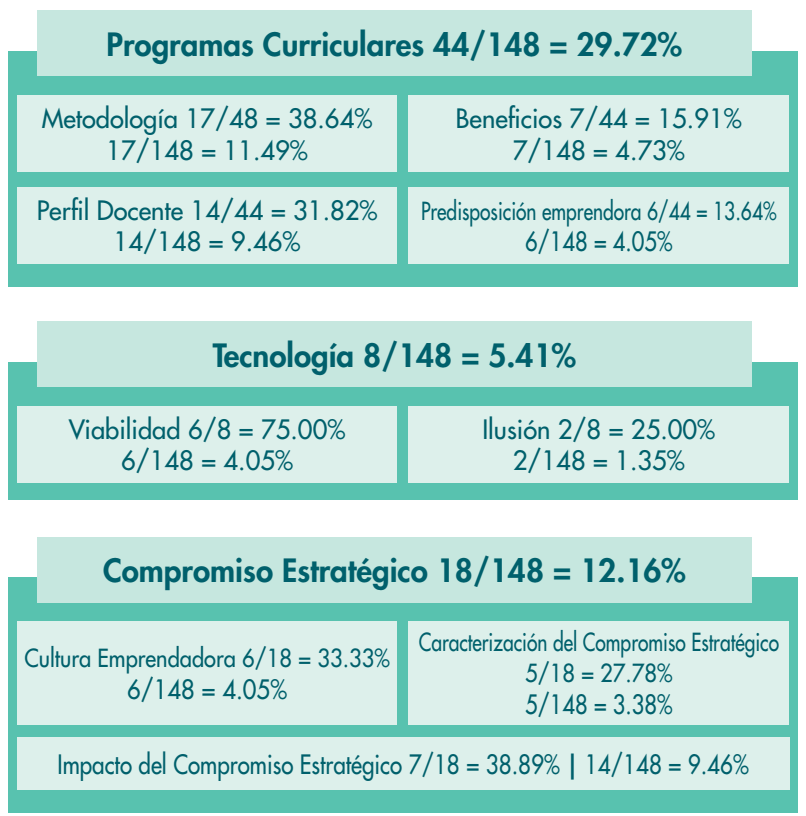

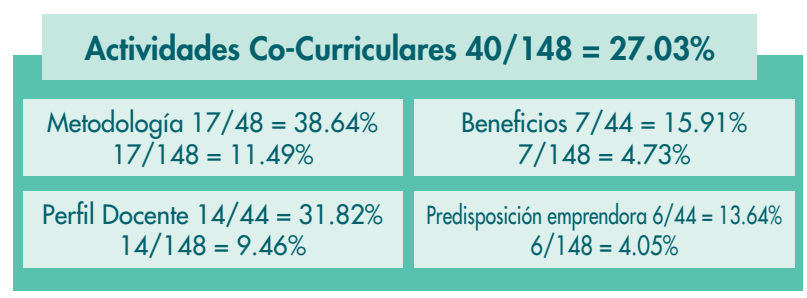

Asignación de Recursos Financieros 16/148 = 10.81\%

$$
\text { Asignación } 16 / 16=100 \%
$$

$16 / 148=10.81 \%$

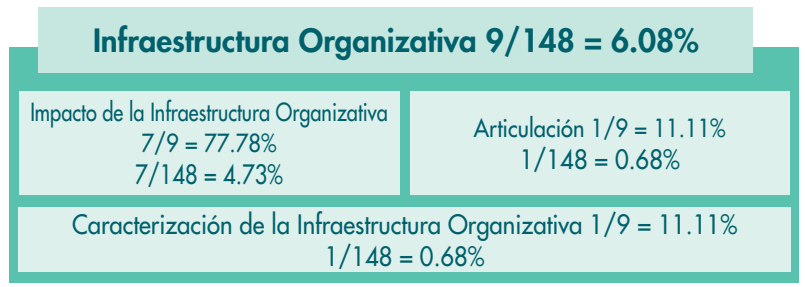

Redes Globales Académicas y de Emprendimiento 13/148 = 8.78\%

Formas de Impacto de las Redes $12 / 13=92.31 \%$ Integración al Sistema Nacional $1 / 13=7.69 \%$

$12 / 148=8.11 \%$

$$
1 / 148=0.68 \%
$$

Nota: Cada tema muestra el valor absoluto de textos afines al mismo y cuánto representan estos textos respecto al total de las 148 citas textuales de las entrevistas. Por otro lado, a lo interno de cada tema, se muestran los subtemas y el número de textos afines que lo componen. Adicionalmente, como referencia, se presenta cuánto representan estos textos del total de su tema y cuánto representan del total de las 148 citas textuales de las entrevistas. 
REFERENCIAS

Acs, Z., Autio, E. \& Szerb, L. (2014). National systems of entrepreneurship: Measurement issues and policy implications. Research Policy, 43(3), 476-494

Acs, Z., Szerb, L., Autio, E. \& Lloyd, A. (2017). The Global Entrepreneurship Index 2017. Global Entrepreneurship Development Institute. https://thegedi.org/globalentrepreneurship-and-development-index/

Acs, Z., Szerb, L., Lafuente, E. \& Lloyd, A. (2018). The Entrepreneurial Ecosystem. In Global Entrepreneurship and Development Index 2018. (pp. 1-9). Springer.

Adekiya, A. \& Ibrahim, F. (2016). Entrepreneurship intention among students. The antecedent role of culture and entrepreneurship training and development. The International Journal of Management Education, 14(2), 116-132.

Ajzen, I. (1991). The theory of planned behavior. Organizational Behavior \& Human Decision Processes, 50(2), 179-211.

Asaka, T. \& Ozeki, K. (Eds.). (1988). Handbook of quality tools, the Japanese approach. Productivity Press.

Audretsch, D., Cunningham, J., Kuratko, D., Lehmann, E. \& Menter, M. (2018). Entrepreneurial ecosystems: economic, technological, and societal impacts. The Journal of Technology Transfer, 44(2), 313-325.

Belitski, M. \& Heron, K. (2017). Expanding entrepreneurship education ecosystems. Journal of Management Development, 36(2), 163-177.

Bell, R. (2015). Developing the next generation of entrepreneurs: Giving students the opportunity to gain experience and thrive. The International Journal of Management Education, 13(1), 37-47.

Brown, R., \& Mason, C. (2017). Looking inside the spiky bits: a critical review and conceptualisation of entrepreneurial ecosystems. Small Business Economics, 49(1), 11-30.

Camacho, D. (2007). Hacia un modelo de emprendimiento universitario. Apuntes del CENES, 275-292.
Guest, G., MacQueen, K. \& Namey, E. (2011). Applied thematic analysis. Sage Publications.

Hakala, H. (2011). Strategic orientations in management literature: Three approaches to understanding the interaction between market, technology, entrepreneurial and learning orientations. International Journal of Management Reviews, 13(2), 199-217.

Hechavarría, D. \& Ingram, A. (2018). Entrepreneurial ecosystem conditions and gendered national-level entrepreneurial activity: a 14-year panel study of GEM. Small Business Economics, 1-28.

Isenberg, D. J. (2010). How to start an entrepreneurial revolution. Harvard Business Review, 88(6), 40-50.

Kantis, H., Federico, J. \& Ibarra, S. (2018). Condiciones sistémicas para el emprendimiento dinámico en América Latina. Cultura, 26, 46.

Kuratko, D., Fisher, G., Bloodgood, J. \& Hornsby, J. (2017). The paradox of new venture legitimation within an entrepreneurial ecosystem. Small Business Economics, 49(1), 119-140.

López, N. \& Sandoval, I. (2016). Métodos y técnicas de investigación cuantitativa y cualitativa. Documento de trabajo. Universidad de Guadalajara. http://recursos.udgvirtual.udg.mx/biblioteca/ bitstream/20050101/1103/1/Metodos_y_tecnicas_de_ investigacion_cuantitativa_y_cualitativa.pdf

Merkens, H. (2004). Selection procedures, sampling, case construction. In Flick, U., von Kardoff, E., \& Steinke, I. (Eds.), A companion to qualitative research. (pp. 231-239). Sage.

Miles, M., Huberman, A. \& Saldaña, J. (2014). Qualitative data analysis: a methods sourcebook. Sage.

Minola, T., Donina, D. \& Meoli, M. (2016). Students climbing the entrepreneurial ladder: Does university internationalization pay off? Small Business Economics, 47(3), 565-587.

Morris, M., Shirokova, G. \& Tsukanova, T. (2017). Student entrepreneurship and the university ecosystem: a multi-country empirical exploration. European Journal of International Management, 11(1), 65-85. 
Nabi, G. \& Holden, R. (2008). Graduate entrepreneurship: intentions, education and training. Education+ training, 50(7), 545-551

Picado, R., Carvajal, J., Sáenz, M. \& Valverde, M. (2015). Universidad e innovación: de la gestión a la acción en el Tecnológico de Costa Rica. Tec Empresarial, 9(2), 19-30.

Rangel, P., Rubiano, M. \& Riaga, C. (2015). Interacción universidad y entorno: marco para el emprendimiento. Educación y educadores, 18(1), 2.

Rice, M., Fetters, M. \& Greene, P. (2014). University-based entrepreneurship ecosystems: a global study of six educational institutions. International Journal of Entrepreneurship and Innovation Management, 18(56), 481-501.

Rideout, E. \& Gray, D. (2013). Does entrepreneurship education really work? A review and methodological critique of the empirical literature on the effects of university-based entrepreneurship education. Journal of Small Business Management, 51(3), 329-351.
Saldaña, J. (2013). The coding manual for qualitative researchers. Sage.

Shirokova, G., Osiyevskyy, O. \& Bogatyreva, K. (2015). Exploring the intention-behavior link in student entrepreneurship: Moderating effects of individual and environmental characteristics. European Management Journal, 34(4), 386-399.

Sieger, P., Fueglistaller, U., Zellweger, T. \& Braun, I. (2019). Global Student Entrepreneurship 2018: Insights From 54 Countries. St. Gallen/Bern: KMU-HSG/IMU.

Stam, F. (2015). Entrepreneurial ecosystems and regional policy: a sympathetic critique. European Planning Studies, 23(9), 1759-1769.

Sussan, F. \& Acs, Z. (2017). The digital entrepreneurial ecosystem. Small Business Economics, 49(1), 55-73.

Wright, M., Siegel, D. \& Mustar, P. (2017). An emerging ecosystem for student start-ups. The Journal of Technology Transfer, 42(4), 909-922. 